\title{
Water distribution network optimisation using HydroGen test instances
}

\author{
A. De Corte \& K. Sörensen \\ University of Antwerp Operations Research Group - ANT/OR, Belgium
}

\begin{abstract}
The water distribution network (WDN) optimisation problem is shown to be a NP-hard problem. Many (metaheuristic) techniques have already been developed in this research area. Despite the aforementioned scientific attention, only a few, high-quality benchmark networks are available for algorithm testing, which, in turn, hinders profound algorithm testing, sensitivity analysis and comparison of the developed techniques. This absence of high-quality benchmark networks motivated us to develop a tool to algorithmically generate close-to-reality virtual WDNs. The tool, called HydroGen, can generate WDNs of arbitrary size and varying characteristics in EPANET or GraphML format. HydroGen is used to generate an extensive library of realistic test networks on which (metaheuristic) methods for the optimisation of WDN design can be tested, allowing researchers in this area to run sensitivity analyses and to draw solid conclusions on the robustness and performance of their methods. An iterated local search technique is developed and tested on a set of Hydrogen-generated water distribution networks.

Keywords: HydroGen, water distribution network design optimisation, iterated local search.
\end{abstract}

\section{Introduction}

Much research has been done on the optimisation of water distribution network design (WDND) over the past thirty years. An overview of developed (metaheuristic) techniques for this challenging optimisation problem is given in [1]. One shortcoming in this research area, as stated in [1], is the lack of adequate testing of the developed optimisation methods. Profound testing is hindered by two factors: (1) the number of available test networks (the so called benchmark networks) is limited and (2) these available test networks do not resemble real 
WDNs. This absence of high-quality test networks motivated us to develop HydroGen [2], a tool that algorithmically generates WDNs of arbitrary size and varying characteristics. A short overview of HydroGen is given in the following Section. In Section 3, a mathematical formulation of the WDND optimisation problem is given. In Section 4, an iterated local search (ILS) technique is presented and used for the optimisation of both existing benchmark networks and HydroGengenerated networks. Experimental results can be found in Section 5. The last Section concludes and provides thoughts for further research.

\section{HydroGen}

Some attempts to construct realistic, virtual WDNs can be found in the literature. The EXNET network [3], Micropolis [4] and Mesopolis [4] are three manually constructed water distribution networks. Modular Design System [5] and WaterNetGen [6] are tools that generate virtual networks algorithmically. A more detailed overview of these methods can be found in [2]. The developed WDN generation method, HydroGen, attempts to address the shortcomings of the aforementioned methods. The tool generates realistic WDNs of arbitrary size and characteristics algorithmically. The networks are available in EPANET input format, since this is the most frequently used hydraulic solver, and GraphML, an XML-based graph exchange file format. A database is available online (http: / / antor.ua.ac.be/download/hydrogen-uw).

The generation method is divided into six phases:

Generation of clusters. In a first step, random cluster centres are generated in a two dimensional plane. The water demand nodes are constructed in a circular layout around these cluster centres, with a uniform random polar angle and a radius for which holds: $P\left[R_{\min } \leq\right.$ radius $\left.\leq \alpha^{d}\left(R_{\max }-R_{\min }\right)\right]=\alpha$, with $\alpha$ being the probability that a generated point will lay within a certain distance from the center and $d$ an adjustable parameter. The generated water demand nodes are not equal to the drinking water needs of individual households, but to clustered demands of households, restaurants, hotels, etc.

Generation of tree structure. A minimum spanning tree, connecting all of the demand nodes in the plane, is drawn, using Prim's algorithm [7]. These connections represent the water distribution pipes. Every pipe has a begin-node, end-node, length, diameter and roughness coefficient. Begin-node, end-node and length are defined by the generation itself: nodes are assigned while drawing the spanning tree and the edge weights or pipe lengths are the corresponding Euclidean distances. Pipes implicitly contain shut-off valves with their initial status set on open.

Addition of reservoirs, tanks and pumps. For every reservoir or tank that has to be added to the WDN, a random demand node at the outside of the cluster is selected, and the water supply is connected to this demand node with a new pipe or pump. Reservoirs are infinite external sources of water to the network. Tanks have 
a limited water storage capacity and operate within their minimum and maximum water levels.

Generation of loops with intra-cluster pipes. Although a tree structure could efficiently provide every demand node with sufficient drinking water, no real-life WDN is designed as a tree. Loops are added to increase water delivery reliability. Moreover, huge pressure changes are avoided by an interwoven net. The user can predefine a cluster type for each cluster. Each cluster type corresponds with a specific meshedness coefficient $M$ ( $M=$ number of actual loops in comparison to the maximum number of loops), which in turn is related to the number of intracluster pipes that is added. Depending on the cluster type, some clusters will have more loops than others, which can be seen in Figure 1. Duplication of pipes and the crossing of pipes are avoided by a preliminary check. Moreover, in reality, the number of neighbouring pipes (or the degree of that node) is limited to four, which is also taken into account in the generation procedure. There are three pre-defined cluster types:

1. rural area $(M=0)$ : no intra-cluster pipes, retaining minimum spanning tree configuration

2. very densely populated area $(0 \ll M)$ : generation of a complete planar graph (via triangulation), followed by adjustments so that the degrees of the nodes are $\leq 4$.

3. urban area $(M=0.16)$ : generation of loops, avoiding duplication and crossing of pipes and maintaining degrees of the nodes $\leq 4$.

Three example networks, with an equal number of nodes but a different cluster type are shown in Figure 1.

Generation of inter-cluster pipes. Additionally, inter-cluster pipes can be added to the WDN. The user can specify the number of inter-cluster pipes that are added randomly, connecting different clusters. In contrast to the intra-cluster pipes, intercluster pipes can cross other pipes.

Assignment of base load and demand patterns. In a final step, both the demand pattern and the base load must be specified for every demand node. Five different user categories with corresponding base loads [8] and demand patterns are defined:

- residential (households): $0.01 \mathrm{~m}^{3} / \mathrm{h}$

- industry (chemical industry, production plants, factories, ... ): $0.17 \mathrm{~m}^{3} / \mathrm{h}$

- commercial (restaurants, cafes, hotels, ... ): $0.20 \mathrm{~m}^{3} / \mathrm{h}$

- public services (hospitals, universities, ... ): $0.48 \mathrm{~m}^{3} / \mathrm{h}$

- energy (refinery, electricity and gas production): $121.68 \mathrm{~m}^{3} / \mathrm{h}$

The water demand of residential users, commercial users and public services is assigned to the pipelines and subsequently clustered to the connected demand nodes, based on the average number of customers per kilometre of pipeline in Flanders (Belgium), which is 43 customers per kilometre of pipeline for residential users [9], 20 commercial users per kilometre and 5 public services buildings per kilometre. The water demand of industrial users and energy plants is assigned to the nodes directly. 

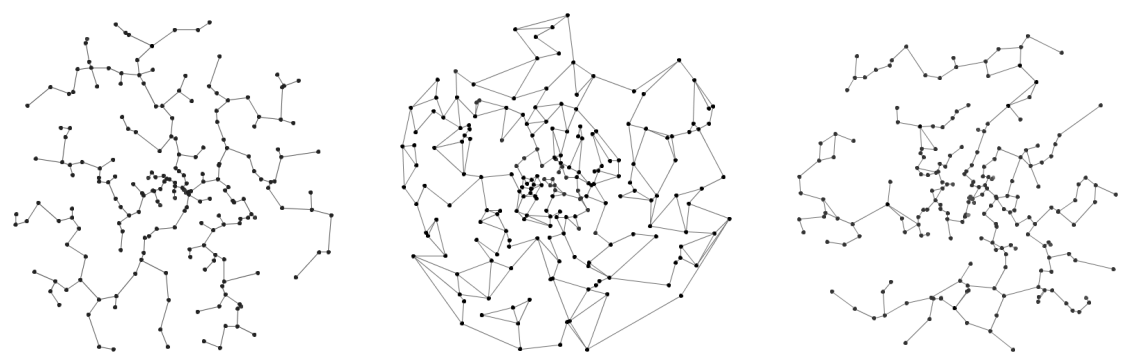

Figure 1: HydroGen examples of: rural area - type 1 (left); very densely populated area $=$ type 2 (center); urban area $=$ type 3 (right).

An abundant number of realistic network settings can be built by adjusting HydroGen's parameters. In [2], it is shown that HydroGen networks show high resemblance to real networks by using a graph-theoretical analysis. This high resemblance is in sharp contrast to the non-realistic benchmark networks and previously developed techniques.

\section{WDND problem formulation}

In this section, we discuss the basic WDND optimisation problem. The aim of this problem is to determine the optimal diameter and material for each pipe in a given network layout. The basic problem is a simplification of reality in that demand for water is considered to be static (or single period), no pumps are assumed to be present in the network (for this reason, these networks are called "gravity-fed") and there is only one objective (cost minimisation).

The objective of this basic WDND optimisation problem is to minimise the total investment cost of the network design. The cost of an individual pipe depends on the type $t$ that is chosen for this pipe from a list of commercially available types $T$. The type of a pipe determines both its diameter and the material of which it is made, which in turn determine its hydraulic properties. If the cost per meter of a pipe $p$ of type $t$ is represented by $I C_{t}$ and the length of pipe $p$ is represented as $L_{p}$, the objective function of the single period, gravity-fed WDND optimisation problem can be written as:

$$
\text { minimize } \sum_{p \in P} \sum_{t \in T} L_{p} I C_{t} x_{p, t} \quad x_{p, t} \in\{0,1\}
$$

where $x_{p, t}$ is a binary decision variable that determines whether pipe $p$ is of type $t$ $\left(x_{p, t}=1\right)$ or not $\left(x_{p, t}=0\right)$.

The objective function is conditioned by physical mass and energy conservation laws, and by minimum head requirements in the demand nodes.

The mass conservation law must be satisfied for each node $n \in N$. This law states that the volume of water flowing into a node in the network per unit of time 
must be equal to the volume of water flowing out of this node. Let $Q_{i n}$ represent the water flowing from node $i$ to node $j$, and let $S_{n}$ be the supply and $D_{n}$ the demand of node $n$ (all expressed in $m^{3} s^{-1}$ ) then the following should hold:

$$
\sum_{i \in N / n} Q_{i n}-\sum_{j \in N / n} Q_{n j}=D_{n}-S_{n} \quad \forall n \in N
$$

Furthermore, for each closed loop $l \in L$, the energy conservation law must be satisfied. This law states that the sum of pressure drops in a closed loop is zero. Pressure drops (also called head losses) in piping systems are caused by wall shear in pipes and friction caused by piping components such as junctions, valves, and bends. In the basic WDND optimisation problem, only the first type of friction losses (in the pipes) are taken into account. For the closed loop $l$, the energy conservation law can therefore be stated as:

$$
\sum_{p \in l} \Delta H_{p}=\sum_{p \in l} \frac{10.6668 y_{p} Q_{p}^{1.852}}{\sum_{t \in T}\left(x_{p, t} C_{t}^{1.852} D_{t}^{4.871}\right)}=0 \quad \forall l \in L \quad y_{p} \in\{-1,1\}
$$

In this equation, head losses in the pipes of the network are approximated using Hazen-Williams equations, with the parameters set to the values used by EPANET2.0, a frequently used hydraulic solver. $y_{p}$ is the sign of $Q_{p}$, which is the water flow rate through pipe $p$ (in $m^{3} s^{-1}$ ), $L_{p}$ is the pipe length (in $m$ ), $C_{t}$ is the Hazen-Williams roughness coefficient of pipe type t(unitless), $D_{t}$ is the diameter of pipe type $t$ (in $m$ ). Parameters $D_{t}$ and $C_{t}$ are determined by the type of a pipe and are assumed given for each available type.

Finally, minimum pressure head requirements exist for every (demand) node $n \in N$. Let $H_{n}$ be the pressure head in node $n$ (in $m$ ) and $H_{n}^{\text {min }}$ the minimum pressure head in node $n$ (in $m$ ). This constraint therefore can be represented as:

$$
H_{n} \geq H_{n}^{\min } \quad \forall n \in N
$$

Several variants of the basic WDND optimisation problem have been proposed. These extensions are more realistic in that the water demand is dynamic (changes during the course of the day), that tanks (with finite capacity) are used in addition to reservoirs for the water supply and that pumps are added to the formerly exclusively gravity-fed networks. Another extension is the formulation of the above single-objective optimisation problem as a multi-objective (where other objectives such as reliability could be added to the cost minimisation objective). In Section 2 can be seen that HydroGen is not only able to generate test networks for the basic WDND optimisation problem, but also for the more complex extensions containing demand patterns, tanks and pumps.

\section{Iterated local search heuristic}

The WDND problem is shown to be NP-hard [10], therefore, metaheuristic techniques are applied to find satisfying solutions in reasonable times. An iterated 


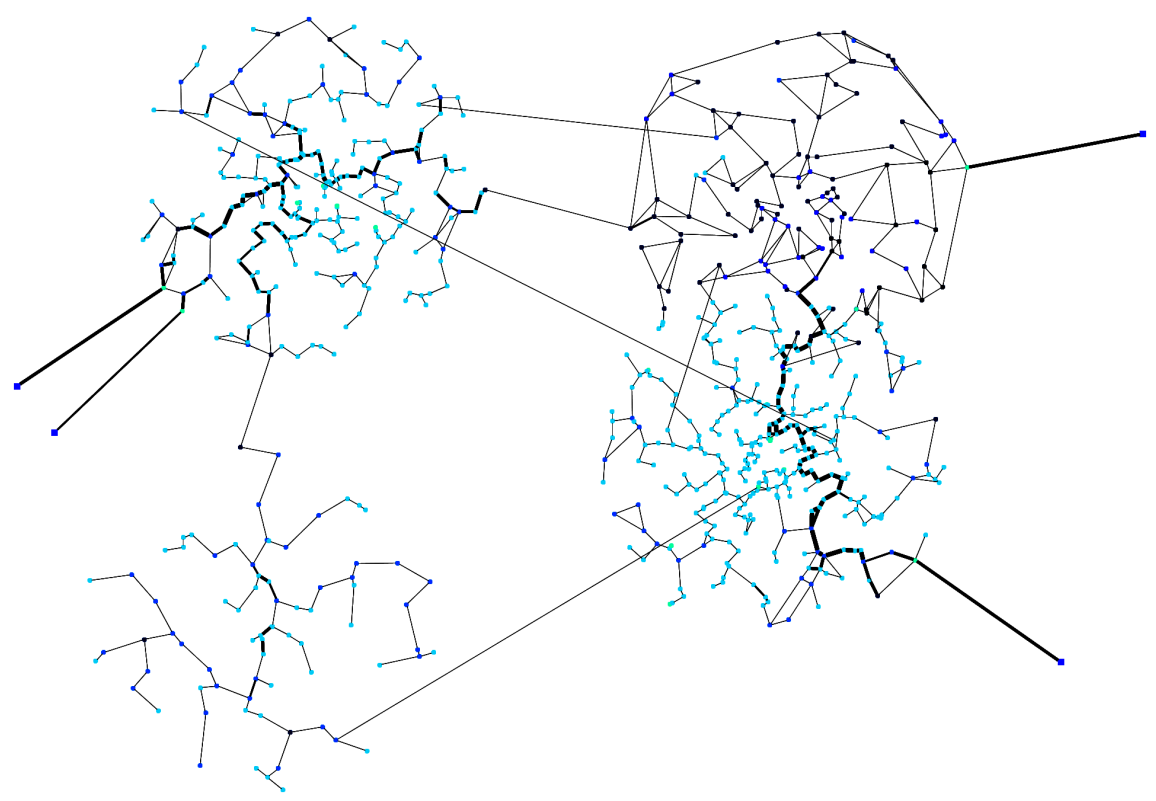

Figure 2: Optimised HydroGen network of 4 clusters: (1) clustertype 1-75 demand nodes, (2) clustertype 2-120 demand nodes, (3) clustertype 3-230 demand nodes, (4) clustertype 3-310 demand nodes.

local search (ILS) algorithm has been developed. ILS iteratively applies a large random change (perturbation) to the current solution, on which the local search algorithm is applied afterwards.

In this work, the basic version of the WDND problem is studied: single-period, gravity-fed, single-objective network design optimisation. ILS iteratively applies a large random change (perturbation) to the current solution, on which the local search algorithm is applied afterwards:

Sort. In a preliminary step, the set of pipes is sorted according to decreasing pipe length and the discrete set of available pipe types is sorted according to decreasing diameter.

Initial solution. In a first step, an initial feasible solution is generated. This solution is the configuration where each pipe is assigned the biggest diameter out of the set of available pipe types, in order to guarantee feasibility.

Local search. The local search algorithm iteratively moves to neighbour solutions by applying a move that tries to decrease the diameter of every pipe with one size. A first improving strategy (adjusting the current solution as soon as a feasible, lower cost solution is encountered) is applied until a local optimum is reached. If 


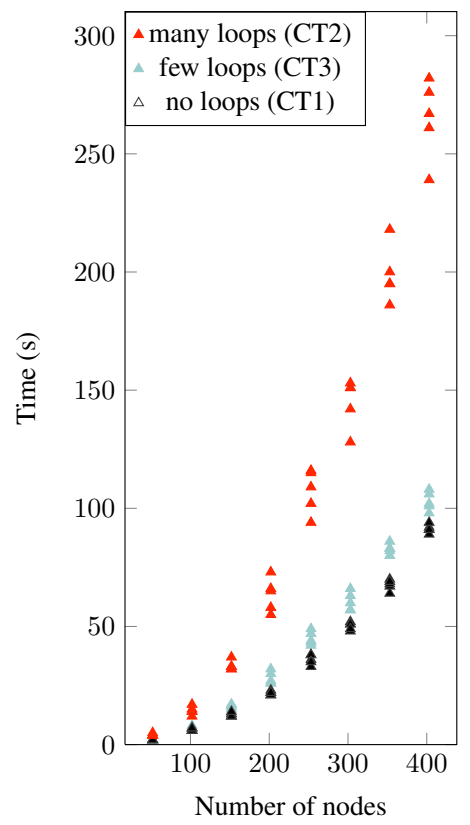

Figure 3: Calculation times of ILS in function of the number of nodes.

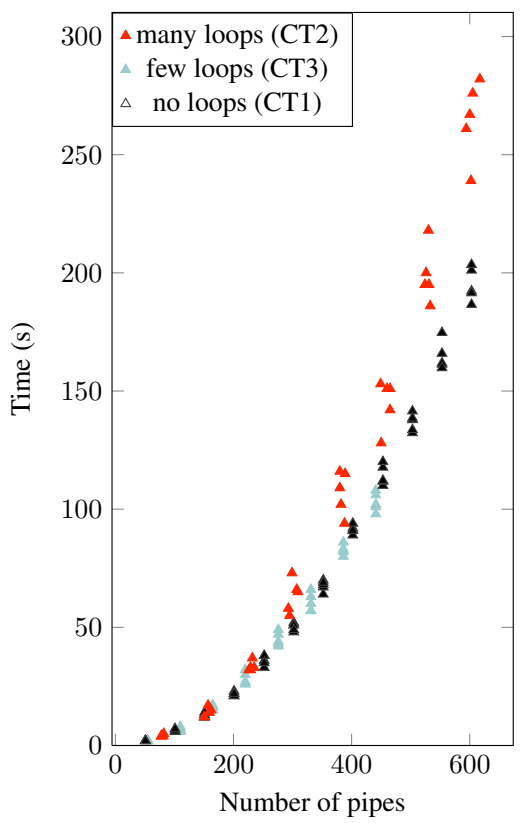

Figure 4: Calculation times of ILS in function of the number of pipes.

this solution is better than the incumbent solution, this new solution replaces the incumbent one as current local optimum.

Perturbation. A perturbation is applied on the current solution to escape local optima. In this perturbation step, for a percentage of randomly selected pipes, diameters are increased with one size. This perturbed solution is the input for the local search algorithm.

Termination criterion. For the current experimental setup, the heuristic stops after 100 iterations.

\section{Experimental results}

The heuristic was tested on the available benchmark networks (New York City Tunnels [11], Hanoi network [12] and Two loop network [13]) and on HydroGen test instances. ILS finds optimal solutions for the available benchmark networks, which confirms the not-too-challenging nature of those networks, as stated in the Introduction.

The HydroGen instances and the set of available pipe types with corresponding costs can be found at the ANT-OR website (http://antor.ua.ac.be/ 
Table 1: Results ILS on HydroGen test instances with $\mathrm{p}=$ number of pipes, $1=$ number of loops, $\mathrm{n}=$ number of nodes. Time is in seconds.

\begin{tabular}{|c|c|c|c|c|c|c|c|c|c|c|c|}
\hline Name & $\mathbf{p}$ & 1 & $\mathbf{n}$ & Cost (avg) & Time & Name & $\mathbf{p}$ & I & $\mathbf{n}$ & Cost (avg) & Time \\
\hline $50-1-1$ & 51 & 0 & 52 & 82,072 & 2 & $50-3-1$ & 55 & 4 & 52 & 64,165 & 2 \\
\hline $50-1-2$ & 51 & 0 & 52 & 93,088 & 2 & $50-3-2$ & 55 & 4 & 52 & 106,161 & 2 \\
\hline $50-1-3$ & 51 & 0 & 52 & 61,020 & 2 & $50-3-3$ & 55 & 4 & 52 & 57,694 & 2 \\
\hline $50-1-4$ & 51 & 0 & 52 & 74,403 & 2 & $50-3-4$ & 55 & 4 & 52 & 93,675 & 2 \\
\hline $50-1-5$ & 51 & 0 & 52 & 72,030 & 2 & $50-3-5$ & 55 & 4 & 52 & 82,623 & 2 \\
\hline $100-1-1$ & 101 & 0 & 102 & 116,101 & 6 & $100-3-1$ & 110 & 9 & 102 & 198,003 & 7 \\
\hline $100-1-2$ & 101 & 0 & 102 & 89,126 & 6 & $100-3-2$ & 110 & 9 & 102 & 98,772 & 8 \\
\hline $100-1-3$ & 101 & 0 & 102 & 129,827 & 6 & $100-3-3$ & 110 & 9 & 102 & 102,945 & 7 \\
\hline $100-1-4$ & 101 & 0 & 102 & 108,089 & 7 & $100-3-4$ & 110 & 9 & 102 & 218,055 & 6 \\
\hline $100-1-5$ & 101 & 0 & 102 & 119,407 & 7 & $100-3-5$ & 110 & 9 & 102 & 119,562 & 6 \\
\hline $150-1-1$ & 151 & 0 & 152 & 124,161 & 13 & $150-3-1$ & 165 & 14 & 152 & 206,937 & 17 \\
\hline $150-1-2$ & 151 & 0 & 152 & 217,761 & 13 & $150-3-2$ & 165 & 14 & 152 & 208,064 & 17 \\
\hline $150-1-3$ & 151 & 0 & 152 & 182,722 & 12 & $150-3-3$ & 165 & 14 & 152 & 193,410 & 16 \\
\hline $150-1-4$ & 151 & 0 & 152 & 119,896 & 12 & $150-3-4$ & 165 & 14 & 152 & 182,723 & 16 \\
\hline $150-1-5$ & 151 & 0 & 152 & 128,207 & 14 & $150-3-5$ & 165 & 14 & 152 & 195,338 & 15 \\
\hline 200-1-1 & 201 & 0 & 202 & 188,806 & 22 & $200-3-1$ & 220 & 19 & 202 & 224,773 & 26 \\
\hline $200-1-2$ & 201 & 0 & 202 & 175,398 & 23 & $200-3-2$ & 220 & 19 & 202 & 246,827 & 26 \\
\hline $200-1-3$ & 201 & 0 & 202 & 157,010 & 21 & $200-3-3$ & 220 & 19 & 202 & 212,997 & 30 \\
\hline $200-1-4$ & 201 & 0 & 202 & 224,879 & 21 & $200-3-4$ & 220 & 19 & 202 & 259,114 & 32 \\
\hline $200-1-5$ & 201 & 0 & 202 & 147,733 & 22 & $200-3-5$ & 220 & 19 & 202 & 249,628 & 27 \\
\hline 250-1-1 & 252 & 0 & 253 & 182,099 & 36 & $250-3-1$ & 276 & 24 & 253 & 222,686 & 47 \\
\hline $250-1-2$ & 252 & 0 & 253 & 192,563 & 38 & $250-3-2$ & 276 & 24 & 253 & 237,939 & 42 \\
\hline $250-1-3$ & 252 & 0 & 253 & 175,797 & 38 & $250-3-3$ & 276 & 24 & 253 & 220,729 & 43 \\
\hline $250-1-4$ & 252 & 0 & 253 & 177,041 & 33 & $250-3-4$ & 276 & 24 & 253 & 207,337 & 49 \\
\hline $250-1-5$ & 252 & 0 & 253 & 196,086 & 35 & $250-3-5$ & 276 & 24 & 253 & 236,656 & 44 \\
\hline $300-1-1$ & 302 & 0 & 303 & 199,627 & 48 & $300-3-1$ & 331 & 29 & 303 & 293,342 & 60 \\
\hline $300-1-2$ & 302 & 0 & 303 & 174,222 & 51 & $300-3-2$ & 331 & 29 & 303 & 249,475 & 57 \\
\hline $300-1-3$ & 302 & 0 & 303 & 157,977 & 52 & $300-3-3$ & 331 & 29 & 303 & 221,862 & 63 \\
\hline $300-1-4$ & 302 & 0 & 303 & 185,115 & 51 & $300-3-4$ & 331 & 29 & 303 & 211,990 & 57 \\
\hline $300-1-5$ & 302 & 0 & 303 & 202,527 & 49 & $300-3-5$ & 331 & 29 & 303 & 275,222 & 66 \\
\hline $350-1-1$ & 352 & 0 & 353 & 249,320 & 70 & $350-3-1$ & 386 & 34 & 353 & 251,396 & 82 \\
\hline $350-1-2$ & 352 & 0 & 353 & 166,115 & 64 & $350-3-2$ & 386 & 34 & 353 & 311,434 & 83 \\
\hline $350-1-3$ & 352 & 0 & 353 & 201,251 & 67 & $350-3-3$ & 386 & 34 & 353 & 212,278 & 80 \\
\hline $350-1-4$ & 352 & 0 & 353 & 180,788 & 68 & $350-3-4$ & 386 & 34 & 353 & 242,706 & 83 \\
\hline $350-1-5$ & 352 & 0 & 353 & 249,730 & 69 & $350-3-5$ & 386 & 34 & 353 & 258,463 & 86 \\
\hline $400-1-1$ & 402 & 0 & 403 & 218,097 & 89 & $400-3-1$ & 441 & 39 & 403 & 297,728 & 101 \\
\hline $400-1-2$ & 402 & 0 & 403 & 241,361 & 92 & $400-3-2$ & 441 & 39 & 403 & 320,679 & 108 \\
\hline $400-1-3$ & 402 & 0 & 403 & 199,615 & 91 & $400-3-3$ & 441 & 39 & 403 & 288,329 & 98 \\
\hline $400-1-4$ & 402 & 0 & 403 & 215,811 & 91 & $400-3-4$ & 441 & 39 & 403 & 234,989 & 102 \\
\hline $400-1-5$ & 402 & 0 & 403 & 184,748 & 94 & $400-3-5$ & 441 & 39 & 403 & 217,215 & 106 \\
\hline $450-1-1$ & 453 & 0 & 454 & 354,389 & 110 & $450-3-1$ & 497 & 44 & 454 & 278,123 & 143 \\
\hline $450-1-2$ & 453 & 0 & 454 & 207,621 & 112 & $450-3-2$ & 497 & 44 & 454 & 256,779 & 141 \\
\hline $450-1-3$ & 453 & 0 & 454 & 207,597 & 118 & $450-3-3$ & 497 & 44 & 454 & 383,187 & 136 \\
\hline $450-1-4$ & 453 & 0 & 454 & 294,367 & 120 & $450-3-4$ & 497 & 44 & 454 & 342,294 & 128 \\
\hline $450-1-5$ & 453 & 0 & 454 & 243,030 & 112 & $450-3-5$ & 497 & 44 & 454 & 211,830 & 133 \\
\hline $500-1-1$ & 503 & 0 & 504 & 253,956 & 138 & $500-3-1$ & 552 & 49 & 504 & 240,588 & 161 \\
\hline $500-1-2$ & 503 & 0 & 504 & 268,311 & 132 & $500-3-2$ & 552 & 49 & 504 & 259,373 & 173 \\
\hline $500-1-3$ & 503 & 0 & 504 & 224,787 & 134 & $500-3-3$ & 552 & 49 & 504 & 255,199 & 177 \\
\hline 500-1-4 & 503 & 0 & 504 & 298,962 & 142 & $500-3-4$ & 552 & 49 & 504 & 249,443 & 169 \\
\hline $500-1-5$ & 503 & 0 & 504 & 245,501 & 139 & $500-3-5$ & 552 & 49 & 504 & 347,346 & 153 \\
\hline
\end{tabular}


Table 1: Continued.

\begin{tabular}{|c|c|c|c|c|c|c|c|c|c|c|c|}
\hline Name & $\mathbf{p}$ & l & $\mathbf{n}$ & Cost (avg) & Time & Name & $\mathbf{p}$ & 1 & $\mathbf{n}$ & Cost (avg) & Time \\
\hline $550-1-1$ & 553 & 0 & 554 & 281,400 & 175 & 550-3-1 & 607 & 54 & 554 & 341,069 & 192 \\
\hline $550-1-2$ & 553 & 0 & 554 & 240,978 & 166 & $550-3-2$ & 607 & 54 & 554 & 322,642 & 211 \\
\hline $550-1-3$ & 553 & 0 & 554 & 214,410 & 161 & $550-3-3$ & 607 & 54 & 554 & 313,738 & 202 \\
\hline $550-1-4$ & 553 & 0 & 554 & 232,131 & 162 & $550-3-4$ & 607 & 54 & 554 & 336,675 & 196 \\
\hline $550-1-5$ & 553 & 0 & 554 & 250,098 & 160 & $550-3-5$ & 607 & 54 & 554 & 307,190 & 200 \\
\hline $600-1-1$ & 603 & 0 & 604 & 295,176 & 187 & & & & & & \\
\hline $600-1-2$ & 603 & 0 & 604 & 310,072 & 192 & & & & & & \\
\hline $600-1-3$ & 603 & 0 & 604 & 223,043 & 192 & & & & & & \\
\hline $600-1-4$ & 603 & 0 & 604 & 248,105 & 201 & & & & & & \\
\hline $600-1-5$ & 603 & 0 & 604 & 203,011 & 204 & & & & & & \\
\hline $50-2-1$ & 78 & 27 & 52 & 517,296 & 4 & $250-2-1$ & 382 & 130 & 253 & $9,834,840$ & 100 \\
\hline $50-2-2$ & 78 & 27 & 52 & 523,516 & 4 & $250-2-2$ & 389 & 137 & 253 & $8,547,468$ & 115 \\
\hline $50-2-3$ & 81 & 30 & 52 & $1,247,833$ & 4 & $250-2-3$ & 380 & 128 & 253 & $10,097,439$ & 109 \\
\hline $50-2-4$ & 78 & 27 & 52 & $2,020,499$ & 4 & $250-2-4$ & 388 & 136 & 253 & $7,631,993$ & 94 \\
\hline $50-2-5$ & 82 & 31 & 52 & 410,842 & 5 & $250-2-5$ & 380 & 128 & 253 & $13,901,087$ & 116 \\
\hline $100-2-1$ & 157 & 56 & 102 & $2,536,095$ & 17 & $300-2-1$ & 449 & 147 & 303 & $15,290,843$ & 153 \\
\hline $100-2-2$ & 150 & 49 & 102 & $1,928,193$ & 12 & $300-2-2$ & 465 & 163 & 303 & $11,079,920$ & 151 \\
\hline $100-2-3$ & 162 & 61 & 102 & $1,793,187$ & 15 & $300-2-3$ & 465 & 163 & 303 & $11,811,356$ & 142 \\
\hline $100-2-4$ & 159 & 58 & 102 & $3,481,872$ & 14 & $300-2-4$ & 460 & 158 & 303 & $12,820,643$ & 151 \\
\hline $100-2-5$ & 158 & 57 & 102 & $2,684,722$ & 14 & $300-2-5$ & 450 & 148 & 303 & $8,924,209$ & 128 \\
\hline $150-2-1$ & 229 & 78 & 152 & $4,647,135$ & 33 & $350-2-1$ & 531 & 179 & 353 & $16,240,748$ & 195 \\
\hline $150-2-2$ & 226 & 75 & 152 & $4,814,073$ & 32 & $350-2-2$ & 530 & 178 & 353 & $19,977,708$ & 218 \\
\hline $150-2-3$ & 229 & 78 & 152 & $3,672,382$ & 32 & $350-2-3$ & 533 & 181 & 353 & $11,680,479$ & 186 \\
\hline $150-2-4$ & 235 & 84 & 152 & $4,486,011$ & 33 & $350-2-4$ & 524 & 172 & 353 & $13,018,354$ & 195 \\
\hline $150-2-5$ & 232 & 81 & 152 & $4,553,862$ & 37 & $350-2-5$ & 526 & 174 & 353 & $13,025,610$ & 200 \\
\hline $200-2-1$ & 309 & 108 & 202 & $8,458,857$ & 65 & $400-2-1$ & 617 & 215 & 403 & $18,863,235$ & 282 \\
\hline $200-2-2$ & 299 & 98 & 202 & $8,074,587$ & 73 & $400-2-2$ & 602 & 200 & 403 & $15,736,287$ & 239 \\
\hline $200-2-3$ & 295 & 94 & 202 & $4,179,127$ & 55 & $400-2-3$ & 600 & 198 & 403 & $22,427,996$ & 267 \\
\hline $200-2-4$ & 307 & 106 & 202 & $5,373,698$ & 66 & $400-2-4$ & 594 & 192 & 403 & $14,343,928$ & 261 \\
\hline $200-2-5$ & 293 & 92 & 202 & $6,141,137$ & 58 & $400-2-5$ & 605 & 203 & 403 & $21,645,310$ & 276 \\
\hline
\end{tabular}

download/hydrogen-uw). A minimal pressure of $30 \mathrm{~m}$ is required in every node. For this experimental set-up, the termination criterion was set at 100 iterations. The perturbation rate is set at 60 percent. Every network was run 10 times, reported data are averages of the results of 10 runs. EPANET version 2.0 was used as hydraulic solver and experiments were done using a personal computer Intel Core i7 with $2.70 \mathrm{GHz}$ processor and $3 \mathrm{~GB}$ Ram.

The ILS algorithm is used to optimise several HydroGen instances. In Table 1, results are presented. Since no other techniques have been applied on these networks yet, comparison is difficult. From Figures 3 and 4, it is clear that, within a certain cluster type, running times increase when the number of nodes (top) or number of pipes increases. Moreover, for a given number of nodes, running times also increase when the networks become more clustered. 


\section{Conclusions and further work}

It has been shown that many (metaheuristic) techniques for WDND optimisation are poorly tested, which makes it difficult to draw solid conclusions on their performance. This lack of rigorous analyses is mainly caused by the absence of high-quality test networks, which motivated us to develop a tool to algorithmically generate realistic, artificial WDNs.The developed tool, HydroGen, generates WDNs of arbitrary size and varying characteristics in EPANET and GraphML format. The use of cluster types; the possibility to add dynamic demand profiles, tanks and pumps and parameter fine-tuning enables HydroGen to generate closeto-reality WDNs. HydroGen is used to generate an extensive library of realistic test networks, which is available via http://antor.ua.ac.be/download/ hydrogen-uw.

An iterated local search metaheuristic has been developed and tested on both benchmark as HydroGen test instances. The heuristic finds optimal results for the benchmark networks, which confirms the not-too-challenging nature of these networks. Results on the HydroGen instances are presented, which will hopefully stimulate researchers in this area to test and compare their techniques on these instances and will allow researcher to draw more solid conclusions on the robustness and performance of their methods.

As stated before, the optimisation of the static, single-period problem could be extended to a multi-period problem with hourly water demand patterns. Pumps could be added to the gravity-fed network setting. Moreover, the single-objective problem could be extended into a multi-objective optimisation problem, where other objectives such as maximisation of network reliability could be taken into account on top of the current cost minimisation goal.

\section{Acknowledgement}

This research was funded by the Research Foundation - Flanders (FWO).

\section{References}

[1] De Corte, A. \& Sörensen, K., Optimisation of gravity-fed water distribution network design: a critical review. European Journal of Operational Research, 228, pp. 1-10, 2013.

[2] De Corte, A. \& Sörensen, K., Hydrogen: an artificial water distribution network generator. Water Resources Management, 2013.

[3] Farmani, R., Savic, D. \& Walters, G., Exnet benchmark problem for multiobjective optimization of large water systems. Proceedings IFAC Workshop, 2004.

[4] Brumbelow, K., Torres, J., Guikema, S., Bristow, E. \& Kanta, L., Virtual cities for water distribution and infrastructure systems research. Proceedings of the World Environmental and Water Resources Congress, Tampa, FL, May, 2007. 
[5] Möderl, M., Sitzenfrei, R., Fetz, T., Fleischhacker, E. \& Rauch, W., Systematic generation of virtual networks for water supply. Water Resources Research, 47, pp. 1-10, 2011.

[6] Muranho, J., Ferreira, A., Sousa, J., Gomes, A. \& Marques, A.S., WaterNetGen: an EPANET extension for automatic water distribution network models generation and pipe sizing. Water Science \& Technology: Water Supply, 12, pp. 117-123, 2012.

[7] Prim, R., Shortest connection networks and some generalizations. Bell System Technical Journal, 36, pp. 1389-1401, 1957.

[8] Van Tomme, I. \& De Sutter, R., Berekening van het watergebruik in 2002 en analyse van het watergebruik in de periode 19991-2002, studie uitgevoerd in opdracht van de vlaamse milieumaatschappij. MIRA, MIRA/200X/06, pp. 1-57, 2004.

[9] VMW, Watermeter 2012: Drinkwaterproductie en -levering in cijfers. Technical report, VMW, 2013.

[10] Yates, D., Templeman, A. \& Boffey, T., The computational complexity of the problem of determining least capital cost designs for water supply networks. Engineering Optimization, 7, pp. 143-155, 1984.

[11] Schaake, J. \& Lai, D., Linear programming and dynamic programming applications to water distribution network design. Report 116, Department of Civil Engineering, MIT, Cambridge, USA, 1969.

[12] Fujiwara, O. \& Khang, D., A two-phase decomposition method for optimal design of looped water distribution networks. Water Resources Research, 26, pp. 539-549, 1990.

[13] Alperovits, A. \& Shamir, U., Design of optimal water distribution systems. Water Resources Research, 13, pp. 885-900, 1977. 